\title{
ON INDEFINITE TERNARY QUADRATIC FORMS
}

\section{R. T. WORLEY}

(Received 24 April 1972; revised 27 November 1972)

Communicated by E. S. Barnes

In a paper [1] with the same title Barnes has shown that if $Q(x, y, z)$ is an indefinite ternary quadratic form of determinant $d \neq 0$ then there exist integers $x_{1}, y_{1}, z_{1}, x_{2}, \cdots, z_{3}$ satisfying

$$
\left|\begin{array}{lll}
x_{1} & y_{1} & z_{1} \\
x_{2} & y_{2} & z_{2} \\
x_{3} & y_{3} & z_{3}
\end{array}\right|= \pm 1
$$

for which

$$
-\frac{2}{3}|d| \leqq Q\left(x_{1}, y_{1}, z_{1}\right) Q\left(x_{2}, y_{2}, z_{2}\right) Q\left(x_{3}, y_{3}, z_{3}\right) \leqq \frac{2}{3}|d|
$$

Furthermore, unless $Q$ is equivalent to a multiple of

$$
Q_{1}(x, y, z)=x^{2}+x y+y^{2}-2 z^{2}
$$

or two other forms $Q_{2}, Q_{3}$ then the constant $\frac{2}{3}$ in (1.2) can be replaced by $1 / 2.2$. For $Q_{1}$ equality is needed on at least one side of (1.2) while for $Q_{2}, Q_{3}$ the constant $\frac{2}{3}$ can be reduced to $12 / 25$ but no further.

As equality is not needed on both sides of (1.2) the question arises as to how small the constant on one side can be while keeping the constant at the other side as $\frac{2}{3}$. The purpose of this paper is to show that one side can be altered to 0 without invalidating (1.2). The precise result obtained is

THEOREM 1. Let $Q$ be an indefinite ternary quadratic form of determinant $d \neq 0$. Then there exist integers $x_{1}, \cdots, z_{3}$ satisfying (1.1) for which

$$
0 \leqq Q\left(x_{1}, y_{1}, z_{1}\right) Q\left(x_{2}, y_{2}, z_{2}\right) Q\left(x_{3}, y_{3}, z_{3}\right) \leqq \frac{2}{3}|d| \text {. }
$$

Furthermore the constant $\frac{2}{3}$ can be improved to $\frac{1462}{2 \frac{379}{37}}=\cdot 616 \cdots$ unless $Q$ is equivalent to a multiple of $Q_{1}$ which requires $z_{3}$. 
It will be noted that, as for the symmetric case, much more can be proved in the case $m(Q)=0$, where $m(Q)$ denotes the lower bound of $|Q(x, y, z)|$ for integral $(x, y, z) \neq(0,0,0)$. In fact we have

THEOREM 2. Let $Q$ be an indefinite ternary quadratic form of non zero determinant with $m(Q)=0$ Then $M_{+}(Q)=0$, where $M_{+}(Q)$ denotes the infimum of the non negative values of

$$
Q\left(x_{1}, y_{1}, z_{1}\right) Q\left(x_{2}, y_{2}, z_{2}\right) Q\left(x_{3}, y_{3}, z_{3}\right)
$$

taken over all sets of integers $x_{1}, \cdots, z_{3}$ satisfying (1.1).

In the proof of theorems 1 and 2 we use the following lemmas.

LEMMA 1. Let $\phi(x, y)$ be a binary quadratic form of discriminant $D>0$ and suppose $\phi$ is not equivalent to a multiple of either of the forms $x^{2}+x y-y^{2}$, $x^{2}-\frac{5}{2} y^{2}$. Then there exist integers $x, y, u, v$ with $|x v-y u|=1$ for which

$$
|\phi(x, y)(u, v)| \leqq \frac{1333}{9797} D .
$$

Lemma 2. Let $k>\frac{1}{2}$ be a real number. Then for any real number $x_{0}$ there exists an integer $x$ for which

$$
\left|\left(x-x_{0}\right)^{2}-k\right| \leqq \begin{cases}1 & \text { if } \frac{1}{2}<k \leqq 2 \\ k-1 & \text { if } 2 \leqq k \leqq 2 \frac{1}{2} \\ 1 \frac{1}{2} & \text { if } 2 \frac{1}{2} \leqq k \leqq 3 \frac{3}{4} \\ \left(k-\frac{1}{4}\right)^{\frac{1}{2}} & \text { for any } k>\frac{1}{2}\end{cases}
$$

Lemma 3. Let $f(x, y, z)=(x+\lambda y+\mu z)^{2}-\phi(y, z)$ be an indefinite ternary quadratic form of non-zero determinant with $m(f) \geqq 1-\varepsilon$. Let $\phi(p, q)=k$ where $p, q$ are relatively prime integers. Then either

$$
k \leqq-\frac{3}{4}+\varepsilon \text {, or } k=\frac{5}{4} \text {, or } k=2 \text {, or } k \geqq 2.21(1-\varepsilon)^{2} .
$$

Further, if $k=\frac{5}{4}$ or 2 then $f(x, p y, q v)$ is equivalent to $x^{2}+x y-y^{2}$ or $x^{2}-2 y^{2}$ respectively.

Lemma 4. Let $\phi(x, y)$ be an indefinite binary quadratic form of discriminant $D>0$ which does not represent zero. Suppose that $\phi(x, y)$ is not equivalent to a multiple of $x^{2}+x y-y^{2}$. Then there exists a form $a x^{2}+b x y+c y^{2}$ equivalent to $\phi(x, y)$ for which $-\frac{5}{32} D \leqq a c<0$ unless $\phi$ is equivalent to a multiple of $x^{2}-3 y^{2}$, when $\phi$ is equivalent to a form $a x^{2}+b x y+c y^{2}$ with $a c=-\frac{1}{6} D$.

Lemma 5. Let $\phi(x, y)$ be an indefinite quadratic form of discriminant $D>0$ which does not represent zero. Suppose that $\phi(x, y)$ is not equivalent to a multiple 
of $x^{2}+k x y-y^{2}$ for $k=1,2,3$ or 4 . Then there exists a form $a x^{2}+b x y+c y^{2}$ equivalent to $\phi(x, y)$ for which $0<a c \leqq \frac{731}{3965} D$.

Lemma 6. Let $a, \lambda, \mu$ be real numbers with $a>1$ and set

$$
F_{\lambda}(x)=(x+\lambda)^{2}-a, F_{\mu}(x)=(x+\mu)^{2}-a .
$$

Then it is possible to find integers $x_{1}, \cdots, x_{4}$ such that

$$
\begin{aligned}
0 & \leqq F_{\lambda}\left(x_{1}\right) F_{\mu}\left(x_{2}\right) \leqq \sigma(a), \\
-\rho(a) & \leqq F_{\lambda}\left(x_{3}\right) F_{\mu}\left(x_{4}\right) \leqq 0
\end{aligned}
$$

where $\sigma(a)$ and $\rho(a)$ are defined as follows. Set $\Delta=2 \sqrt{ } a$ and let $n$ be the integer such that $n-1<\Delta \leqq n$. Let

Then

$$
\begin{aligned}
& \sigma_{1}(a)=\frac{1}{16} \min \left\{\left(n^{2}-\Delta^{2}\right)^{2},\left(\Delta^{2}-(n-2)^{2}\right)^{2}\right\}, \\
& \sigma_{2}(a)=\frac{1}{16} \min \left\{\left(n^{2}-\Delta^{2}\right)\left((n+1)^{2}-\Delta^{2}\right),\right. \\
& \left.\quad\left(\Delta^{2}-(n-1)^{2}\right)\left(\Delta^{2}-(n-2)^{2}\right)\right\}, \\
& \sigma_{3}(a)=\frac{1}{16}\left(\Delta^{2}-(n-1)^{2}\right)^{2}, \\
& \rho_{1}(a)=\frac{1}{16}\left(n^{2}-\Delta^{2}\right)\left(\Delta^{2}-(n-2)^{2}\right), \\
& \rho_{2}(a)=\frac{1}{16}\left((n+1)^{2}-\Delta^{2}\right)\left(\Delta^{2}-(n-1)^{2}\right) .
\end{aligned}
$$

$$
\begin{aligned}
& \sigma(a)=\max \left(\sigma_{1}(a), \sigma_{2}(a), \sigma_{3}(a)\right), \text { and } \\
& \rho(a)=\max \left(\rho_{1}(a), \rho_{2}(a)\right) .
\end{aligned}
$$

Lemma 7. Let $a>2$. Then if $\sigma(a), \rho(a)$ are defined as in lemma 6 we have

$$
\begin{aligned}
& a^{-2} \sigma(a) \leqq 18 / 49 \\
& a^{-2} \rho(a) \leqq 9 / 16
\end{aligned}
$$

LEMMA 8. Let $\phi(y, z)$ be a non-zero indefinite binary quadratic form of discriminant $D>0$. Let $\rho$ be a real number satisfying $0<\rho<1$. Then $\phi$ is equivalent to a form $a y^{2}+b y z+c z^{2}$ where either

(i) $a y^{2}+b y z+c z^{2}$ is reduced ${ }^{*}$ and $a \geqq \rho \sqrt{ } D$, or

(ii) $0<a<\rho \sqrt{ } D, \rho \sqrt{ } D<c<\sigma \sqrt{ } D$ where $\sigma=2 \rho+\sqrt{ }\left(1+4 \rho^{2}\right)$.

Of these lemmas, only the last three need to be proved here, as the first four are to be found in the paper of Barnes mentioned earlier, while the fifth is a partial restatement of the work of Barnes [2].

* See e.g. L.E. Dickson [3] p. 80. A form $a x^{2}+b x y+c y^{2}$ with $a>0$ is reduced if $b>0$, $c<0$ and $0<\Delta-b<2|c|<\Delta+b$ where $\Delta=\sqrt{ }\left(b^{2}-4 a c\right)$. 
Proof of Lemma 6. It is clear that we may take $|\lambda| \leqq \frac{1}{2}$ and $|\mu| \leqq \frac{1}{2}$, indeed by changing the sign of $x$ if necessary we may take $0 \leqq \lambda \leqq \frac{1}{2}, 0 \leqq \mu \leqq \frac{1}{2}$. We assume first that $n$ is even. From the definition of $n$ we have

$$
\begin{aligned}
0 \leqq F_{\lambda}\left(\frac{1}{2} n\right) & \leqq \frac{1}{4}\left((n+1)^{2}-\Delta^{2}\right), \\
-\frac{1}{4}\left(\Delta^{2}-(n-2)^{2}\right) & \leqq F_{\lambda}\left(\frac{1}{2} n-1\right)<0, \\
-\frac{1}{4}\left(\Delta^{2}-(n-1)^{2}\right) & \leqq F_{\lambda}\left(-\frac{1}{2} n\right) \leqq \frac{1}{4}\left(n^{2}-\Delta^{2}\right)
\end{aligned}
$$

and similarly for $F_{\mu}$. We consider three cases.

(a) $F_{\lambda}\left(-\frac{1}{2} n\right), F_{\mu}\left(-\frac{1}{2} n\right)$ both positive. In this case

$$
\begin{aligned}
0<F_{\lambda}\left(\frac{1}{2} n-1\right) F_{\mu}\left(\frac{1}{2} n-1\right) & \leqq \frac{1}{16}\left(\Delta^{2}-(n-2)^{2}\right)^{2}, \\
0<F_{\lambda}\left(-\frac{1}{2} n\right) F_{\mu}\left(-\frac{1}{2} n\right) & \leqq \frac{1}{16}\left(n^{2}-\Delta^{2}\right), \text { and } \\
-\frac{1}{16}\left(n^{2}-\Delta^{2}\right)\left(\Delta^{2}-(n-2)^{2}\right) & \leqq F_{\lambda}\left(-\frac{1}{2} n\right) F_{\mu}\left(\frac{1}{2} n-1\right)<0 .
\end{aligned}
$$

(b) $F_{\lambda}\left(-\frac{1}{2} n\right), F_{\mu}\left(-\frac{1}{2} n\right)$ of opposite sign or at least one zero. We assume $F_{\mu}\left(-\frac{1}{2} n\right) \leqq 0$ without loss of generality. Then either $F_{\lambda}\left(-\frac{1}{2} n\right) F_{\mu}\left(-\frac{1}{2} n\right)=0$ or

$$
\begin{aligned}
& 0 \leqq F_{\mu}\left(-\frac{1}{2} n\right) F_{\lambda}\left(\frac{1}{2} n-1\right) \leqq \frac{1}{16}\left(\Delta^{2}-(n-1)^{2}\right)\left(\Delta^{2}-(n-2)^{2}\right), \\
& 0 \leqq F_{\lambda}\left(-\frac{1}{2} n\right) F_{\mu}\left(\frac{1}{2} n\right) \leqq \frac{1}{16}\left(n^{2}-\Delta^{2}\right)\left((n+1)^{2}-\Delta^{2}\right), \text { and } \\
&-\frac{1}{16}\left(n^{2}-\Delta^{2}\right)\left(\Delta^{2}-(n-1)^{2}\right) \leqq F_{\lambda}\left(-\frac{1}{2} n\right) F_{\mu}\left(-\frac{1}{2} n\right) \leqq 0 .
\end{aligned}
$$

(c) $F_{\lambda}\left(-\frac{1}{2} n\right), F_{\mu}\left(-\frac{1}{2} n\right)$ both negative. In this case

$$
\begin{aligned}
& 0<F_{\lambda}\left(-\frac{1}{2} n\right) F_{\mu}\left(-\frac{1}{2} n\right) \leqq \frac{1}{16}\left(\Delta^{2}-(n-1)^{2}\right)^{2} \text { and } \\
& -\frac{1}{16}\left((n+1)^{2}-\Delta^{2}\right)\left(\Delta^{2}-(n-1)^{2}\right) \leqq F_{\lambda}\left(-\frac{1}{2} n\right) F_{\mu}\left(\frac{1}{2} n\right)<0 .
\end{aligned}
$$

Clearly in each case it is possible to choose integers $x_{1}, \cdots, x_{4}$ satisfying (1.3) and (1.4).

If $n$ is odd we repeat the above analysis with $\lambda, \mu$ replaced by $\frac{1}{2}-\lambda, \frac{1}{2}-\mu$ respectively to obtain integers $x_{1}, \cdots, x_{4}$ satisfying

$$
0 \leqq\left\{\left(-\left(x_{1}+\frac{1}{2}\right)+\left(\frac{1}{2}-\lambda\right)\right)^{2}-a\right\}\left\{\left(-\left(x_{2}+\frac{1}{2}\right)+\left(\frac{1}{2}-\mu\right)\right)^{2}-a\right\} \leqq \sigma(a),
$$

and

$$
-\rho(a) \leqq\left\{\left(-\left(x_{3}+\frac{1}{2}\right)+\left(\frac{1}{2}-\lambda\right)\right)^{2}-a\right\}\left\{\left(-\left(x_{4}+\frac{1}{2}\right)+\left(\frac{1}{2}-\mu\right)\right)^{2}-a\right\} \leqq 0,
$$

which are, in fact, (1.3) and (1.4).

Proof OF Lemma 7. It should first be observed that $n \geqq 3$ since $a>2$, and that $n \geqq 4$ for $a>2.25$. We consider $\sigma_{1}, \sigma_{2}, \sigma_{3}$, and $\rho$ separately

(a) For $2<a \leqq 2.25$ we have $a^{-2} \sigma_{1}(a)=\Delta^{-4}\left(n^{2}-\Delta^{2}\right)^{2} \leqq \frac{1}{64}$. For $a>2 \cdot 25$ it is easily verified that 


$$
a^{-2} \sigma_{1}(a)=\Delta^{-4}\left(\Delta^{2}-(n-2)^{2}\right)^{2} \leqq 4(n-1)\left(n^{2}-2 n+2\right)^{-2}
$$

for $(n-1)^{2}<\Delta^{2} \leqq(n-1)^{2}+1$, and

$$
a^{-2} \sigma_{1}(a)=\Delta^{-4}\left(n^{2}-\Delta^{2}\right)^{2} \leqq 4(n-1)^{2}\left(n^{2}-2 n+2\right)^{-2}
$$

for $(n-1)^{2}+1 \leqq \Delta^{2} \leqq n^{2}$. As $(n-1)^{2}<\Delta^{2} \leqq n^{2}$ from the definition of $n$, as $n \geqq 4$, and as the right hand side of the above two inequalities increases with $n$ we clearly have $a^{-2} \sigma_{1}(a) \leqq 36 / 100$.

(b) In a similar manner we have

$$
a^{-2} \sigma_{2}(a)=\Delta^{-4}\left(\Delta^{2}-(n-2)^{2}\right)\left(\Delta^{2}-(n-1)^{2}\right) \leqq 3 n(n-1)\left(n^{2}-n+1\right)^{-2}
$$

for $\Delta^{2} \leqq n^{2}-n+1$, and

$$
a^{-2} \sigma_{2}(a)=\Delta^{-4}\left((n+1)^{2}-\Delta^{2}\right)\left(n^{2}-\Delta^{2}\right) \leqq 3 n(n-1)\left(n^{2}-n+1\right)^{-2}
$$

for $\Delta^{2} \geqq n^{2}-n+1$. Thus, since $n \geqq 3$ we clearly obtain the inequality $a^{-2} \sigma_{2}(a) \leqq 18 / 49$.

(c) Similarly

$$
a^{-2} \sigma_{2}(a)=\Delta^{-4}\left(\Delta^{2}-(n-1)^{2}\right)^{2} \leqq n^{-4}\left(n^{2}-(n-1)^{2}\right)^{2}
$$

since $n-1<\Delta \leqq n$. Hence $a^{-2} \sigma_{3}(a) \leqq 25 / 81$ since $n \geqq 3$.

Thus we have

$$
a^{-2} \sigma(a) \leqq \max (1 / 64,36 / 100,18 / 49,25 / 81)=18 / 49 .
$$

(d) It is simple to verify that

$$
a^{-2}\left(\rho_{1}(a)-\rho_{2}(a)\right)=\Delta^{-4}(2 n-1)\left(\left(2 n^{2}-2 n-1\right)-2 \Delta^{2}\right) .
$$

Thus for $\Delta^{2} \leqq n^{2}-n-\frac{1}{2}$ we have

$$
a^{-2} \rho(a)=a^{-2} \rho_{1}(a)=\left(n^{2} \Delta^{-2}-1\right)\left(1-(n-2)^{2} \Delta^{-2}\right) .
$$

Treating $\Delta^{2}$ as a variable satisfying $(n-1)^{2}<\Delta^{2} \leqq n^{2}-n-\frac{1}{2}$ it is easily checked that the right hand side of the above equation is always less than the value at $\Delta^{2}=(n-1)^{2}$, the expression being decreasing in the allowable range. Thus

$$
a^{-2} \rho(a)<(2 n-1)(2 n-3)(n-1)^{-4} \leqq 35 / 81
$$

since $n^{2}-n-\frac{1}{2} \geqq \Delta^{2}>8$ implies that $n \geqq 4$. For $\Delta^{2}>n^{2}-n+\frac{1}{2}$ we have

$$
a^{-2} \rho(a)=\Delta^{-4}\left((n+1)^{2}-\Delta^{2}\right)\left(\Delta^{2}-(n-1)^{2}\right),
$$

and analysis shows this to have a maximum of $4 n^{2}\left(n^{2}-1\right)^{-2}$ when $\Delta^{2}=\left(n^{2}-1\right)^{2} /\left(n^{2}+1\right)$. Since $n \geqq 3$ it follows that $a^{-2} \rho(a) \leqq 9 / 16$. Thus for both ranges of $\Delta^{2}$ we have $a^{-2} \rho(a) \leqq 9 / 16$, as desired. 
Proof of Lemma 8. Let $a y^{2}+b y z+c z^{2}$ be a reduced form equivalent to $\phi$ with $a>0$. We assume $a<\rho \sqrt{ } D$ for otherwise (i) is valid. The reduction conditions imply that $a+b+c>0$ and so in the equivalent form

$$
a y^{2}+(b+2 a) y z+(a+b+c) z^{2}=a y^{2}+b^{\prime} y z+c^{\prime} z^{2}
$$

we have $c^{\prime}>0$. Plainly $c^{\prime}<a+b<(\rho+1) \sqrt{ } D<\sigma \sqrt{ } D$, so either this form satisfies (ii) or $c^{\prime}<\rho \sqrt{ } D$. In the latter case we have in the equivalent form

$$
a y^{2}+\left(b^{\prime}+2 a\right) y z+\left(a+b^{\prime}+c^{\prime}\right) z^{2}=a y^{2}+b^{\prime \prime} y z+c^{\prime \prime} z^{2}
$$

that $c^{\prime \prime}>b^{\prime}>\left(\left(b^{\prime}\right)^{2}-4 a c^{\prime}\right)^{\frac{1}{2}}=\sqrt{ } D>\rho \sqrt{ } D$, and

$$
c^{\prime \prime}=a+\left(D+4 a c^{\prime}\right)^{\frac{1}{2}}+c^{\prime} \leqq\left(\rho+\left(1+4 \rho^{2}\right)^{\frac{1}{2}}+\rho\right) \sqrt{ } D=\sigma \sqrt{ } D .
$$

In this case we have found a form for which (ii) holds.

It will be noted that if $k$ is a positive integer and $\rho=\left(k^{2}+4 k\right)^{-\frac{1}{2}}$ then the form $x^{2}+k x y-k y^{2}$ takes no positive values between $1=\rho \sqrt{ } D$ and $k+4=\sigma \sqrt{ } D$. For related work consult [4].

The Proof of Theorem 2. If the lower bound $m(Q)=0$ is attained then there exist relatively prime integers $x_{1}, y_{1}, z_{1}$, such that $Q\left(x_{1}, y_{1}, z_{1}\right)=0$. Choosing integers $x_{2}, \cdots, z_{3}$ to satisfy $(1.1)$ it is clear that $M_{+}(Q)=0$. We therefore assume $m(Q)=0$ is not attained, when by a result of Oppenheim [5] $Q$ takes arbitrarily small values of either sign. Considering $-Q$ if necessary we may assume that $Q$ has signature 1 and that $Q$ takes arbitrarily small positive values. Then for arbitrarily small $\varepsilon_{0}<\frac{1}{5}|d|^{\frac{1}{2}}$ where $d=\operatorname{det}(Q)$ we can choose relatively prime integers $x_{0}, y_{0}, z_{0}$ such that $Q\left(x_{0}, y_{0}, z_{0}\right)=\varepsilon^{2}$ where $0<\varepsilon^{2}<\varepsilon_{0}$. Indeed we may assume $Q(1,0,0)=\varepsilon^{2}$ on applying a suitable integral unimodular transformation. We write

$$
Q(x, y, z)=\varepsilon^{2}(x+\lambda y+\mu z)^{2}-\phi(y, z)
$$

where $\phi$ is an indefinite binary quadratic form, and set

$$
\begin{aligned}
& \phi=a y^{2}+b y z+c z^{2}, \\
& D=b^{2}-4 a c=4 d \varepsilon^{-2} .
\end{aligned}
$$

We suppose firstly that $\phi$ represents zero. By means of a suitable transformation we may take $\phi= \pm \sqrt{ } D y z+c z^{2}$, and after a further transformation of the type $y \rightarrow y+p z, z \rightarrow z$ which replaces $c$ by $c \pm p \sqrt{ } D$ we may assume $\sqrt{ } D \leqq c$ $<2 \sqrt{ } D$. The inequalities on $c, \varepsilon$ and $\varepsilon_{0}$ ensure $c>\varepsilon^{2}$, so we can choose integers $x_{3}, x_{3}^{\prime}$ to satisfy

$$
\varepsilon^{-1} \sqrt{ } c<x_{3}+\mu<2 \varepsilon^{-1} \sqrt{ } c, \varepsilon^{-1} \sqrt{ } c-1 \leqq x_{3}^{\prime}+\mu<\varepsilon^{-1} \sqrt{ } c
$$


respectively. Hence

$$
M_{+}(Q) \leqq Q(1,0,0) Q(0,1,0) Q\left(x_{3}, 0,1\right) \leqq \varepsilon^{2} \cdot \frac{1}{4} \varepsilon^{2} \cdot\left\{\varepsilon^{2}\left(2 \varepsilon^{-1} \sqrt{ } c\right)^{2}-c\right\}=\frac{1}{4} \varepsilon^{4} 3 c .
$$

Thus as $\varepsilon c$ is bounded above independently of $\varepsilon$ we have $M_{+}(Q) \ll \varepsilon^{3}$. Similarly

$$
\begin{aligned}
M_{+}(-Q) \leqq-Q(1,0,0) & Q(0,1,0) Q\left(x_{3}^{\prime}, 0,1\right) \\
& \leqq \varepsilon^{2} \cdot \frac{1}{4} \varepsilon^{2}\left\{c-\varepsilon^{2}\left(\varepsilon^{-1} \sqrt{ } c-1\right)^{2}\right\}=\frac{1}{4} \varepsilon^{4}\left(2 \varepsilon \sqrt{ } c-\varepsilon^{2}\right)
\end{aligned}
$$

Hence $M_{+}(-Q) \ll \varepsilon^{9 / 2}$.

We now suppose $\phi$ does not represent zero. By lemma 8 we may assume $\phi$ to be such that either

(i) $\frac{1}{10} \sqrt{ } D \leqq a \leqq \sqrt{ } D,-\sqrt{ } D \leqq c<0$, or

(ii) $0<a<\frac{1}{10} \sqrt{ } D, \frac{1}{10} \sqrt{ } D \leqq c<\frac{12}{10} \sqrt{ } D$.

In the first case we have $a>\varepsilon^{2}$ and choosing $x_{2}, x_{2}^{\prime}, x_{3}$ to satisfy

$$
\varepsilon^{-1} \sqrt{a}<x_{2}+\lambda \leqq \varepsilon^{-1} \sqrt{a}+1, \varepsilon^{-1} \sqrt{a}-1 \leqq x_{2}^{\prime}+\lambda<\varepsilon^{-1} \sqrt{a},\left|x_{3}+\mu\right| \leqq \frac{1}{2}
$$

respectively we have

$$
M_{+}(Q) \leqq Q(1,0,0) Q\left(x_{2}, 1,0\right) Q\left(x_{3}, 0,1\right) \leqq \varepsilon^{2} \cdot \varepsilon^{2}\left(2 \varepsilon^{-1} \sqrt{ } a+1\right)\left(\frac{1}{4} \varepsilon^{2}+|c|\right) .
$$

Thus $M_{+}(Q) \ll \varepsilon^{\frac{3}{2}}$ as $\sqrt{a} \ll \varepsilon^{-\frac{1}{2}}$ and $|c| \ll \varepsilon^{-1}$. Similarly

$$
M_{+}(-Q) \leqq Q(1,0,0) Q\left(x_{2}^{\prime}, 1,0\right) Q\left(x_{3}, 0,1\right) \ll \varepsilon^{\frac{3}{2}} .
$$

In the second case we can choose $x_{2}$ to make $Q\left(x_{2}, 1,0\right)$ positive and $\ll \varepsilon^{\frac{1}{2}}$ and $x_{3}$ to make $Q\left(x_{3}, 0,1\right)$ of either sign such that $\left|Q\left(x_{3}, 0,1\right)\right| \ll \varepsilon^{\frac{1}{2}}$, and so both $M_{+}(Q) \ll \varepsilon^{3}$ and $M_{+}(-Q) \ll \varepsilon^{3}$.

Hence in all cases we have $M_{+}(Q) \ll \varepsilon$ and $M_{+}(-Q) \ll \varepsilon$. Since $\varepsilon>0$ can be made arbitrarily small we therefore have $M_{+}(Q)=0$ and $M_{+}(-Q)=0$.

We now examine the special form $Q_{1}$. For this form Barnes has shown that the product

$$
Q_{1}\left(x_{1}, y_{1}, z_{1}\right) Q_{1}\left(x_{2}, y_{2}, z_{2}\right) Q_{1}\left(x_{3}, y_{3}, z_{3}\right)
$$

is at least $1=\frac{2}{3}|d|$ in absolute value for integers $x_{1}, \cdots, z_{3}$ satisfying (1.1). Since $Q_{1}(1,0,0)=1, Q_{1}(0,1,0)=1, Q_{1}(1,1,1)=1$ and $Q_{1}(1,0,1)=-1$ it is clear that (3.1) can be made both +1 and -1 . Thus $M_{+}\left(Q_{1}\right)=1=\frac{2}{3}|d|$ and $M_{+}\left(-Q_{1}\right)=1=\frac{2}{3}|d|$. 
The Proof of Theorem 1. We may suppose that $Q$ is an indefinite ternary quadratic form of determinant $d \neq 0$ and that $m(Q) \neq 0$. In fact, since $|d|^{-1} M_{+}(Q),|d|^{-1} M_{+}(-Q)$ are unchanged on multiplying $Q$ by a positive constant and interchanged on multiplying by a negative constant we may take $m_{+}(Q)$ $=m(Q)=1$ where $m_{+}(Q)$ is the lower bound of non-negative values of $Q$. Then for any sufficiently small $\varepsilon_{0}, 0 \leqq \varepsilon_{0}<1$ there exists $\varepsilon, 0 \leqq \varepsilon<\varepsilon_{0}$ and relatively prime integers $x_{0}, y_{0}, z_{0}$ such that $Q\left(x_{0}, y_{0}, z_{0}\right)=1 /(1-\varepsilon)$. After applying a suitable integral unimodular transformation we may suppose $\left(x_{0}, y_{0}, z_{0}\right)=(1,0,0)$ and set

$$
f(x, y, z)=(1-\varepsilon) Q(x, y, z)=(x+\lambda y+\mu z)^{2}-\phi(y, z),
$$

where $\phi(y, z)=a y^{2}+b y z+c z^{2}$ has discriminant

$$
D=b^{2}-4 a c=-4 d(1-\varepsilon)^{3}=-4 d_{1} .
$$

We discuss the cases $\phi$ indefinite, $\phi$ definite, separately, and show that if $f$ is not a multiple of $Q_{1}$ then both $\left|d_{1}\right|^{-1} M_{+}(f)<k$ and $\left|d_{1}\right|^{-1} M_{+}(-f)<k$, where $k=\frac{1472}{2379}=\cdot 616 \cdots$.

Suppose firstly that $\phi$ is indefinite. In this case we assume that $f$ does not take the value -1 , since if it did we could replace $f$ by $-f$ of the desired shape with $\phi$ definite and apply the argument to be used later. By lemma 3, therefore, $\phi$ takes no values in $\left(-\frac{3}{4}+\varepsilon, 2.21(1-\varepsilon)^{2}\right)$, and lemma 4 gives four possibilities to discuss.

(a) $\phi(y, z)$ is equivalent to a multiple of $y^{2}+y z-z^{2}$. We may take this multiple to be positive since $\phi$ is equivalent to its negative, and write

$$
f(x, y, z)=(x+\lambda y+\mu z)^{2}-a\left(y^{2}+y z-z^{2}\right)
$$

where $a \geqq 2.21(1-\varepsilon)^{2}$. In this case

$$
f(1,0,0) f\left(x_{2}, 1,0\right) f\left(x_{3}, 1,1\right)=\left\{\left(x_{2}+\lambda\right)^{2}-a\right\}\left\{\left(x_{3}+\lambda+\mu\right)^{2}-a\right\}
$$

and so by lemma 6 we have $M_{+}(f) \leqq \sigma(a), M_{+}(-f) \leqq \rho(a)$. Since $\left|d_{1}\right|=\frac{5}{4} a^{2}$ we have, by lemma 7 , that

$$
M_{+}(f) \leqq \frac{72}{245}\left|d_{1}\right|, M_{+}(-f) \leqq \frac{9}{20}\left|d_{1}\right|
$$

provided $\varepsilon_{0}$ is sufficiently small.

(b) $\phi(y, z)$ is equivalent to a positive multiple of $y^{2}-3 z^{2}$. We write

$$
f(x, y, z)=(x+\lambda y+\mu z)^{2}-a\left(y^{2}-3 z^{2}\right)
$$

where $a \geqq 2.21(1-\varepsilon)^{2}$. In this case

$$
\left.f(1,0,0) f\left(x_{2}, 1,0\right) f\left(x_{3}, 2,1\right)=\left\{(x+\lambda)^{2}-a\right\}\left\{(x+2 \lambda+\mu)^{2}-a\right)\right\}
$$


and the argument of (a) shows that $M_{+}(f) \leqq \frac{6}{49}\left|d_{1}\right|, M_{+}(-f) \leqq \frac{3}{16}\left|d_{1}\right|$ since $\left|d_{1}\right|=3 a^{2}$.

(c) $\phi(y, z)$ is equivalent to a negative multiple of $y^{2}-3 z^{2}$. We write, since $y^{2}-3 z^{2} \sim-\left(2 y^{2}+2 y z-z^{2}\right)$,

$$
f(x, y, z)=(x+\lambda y+\mu z)^{2}-a\left(2 y^{2}+2 y z-z^{2}\right)
$$

where $a \geqq 1 \cdot 105$. By lemmas 4 and 5 , since $f$ does not take the value -1 , we have the existence of integers $x_{1}, x_{1}^{\prime}, y_{1}, y_{1}^{\prime}, x_{2}, \cdots, y_{2}^{\prime}$, for which

such that

$$
\left|x_{1} y_{2}-x_{2} y_{1}\right|=\left|x_{1}^{\prime} y_{2}^{\prime}-x_{2} y_{1}^{\prime}\right|=1
$$

and

$$
0<f\left(x_{1}, y_{1}, 0\right) f\left(x_{2}, y_{2}, 0\right) \leqq \frac{731}{3965} \cdot 8 a
$$

$$
-\frac{1}{6} \cdot 8 a \leqq f\left(x_{1}^{\prime}, y_{1}^{\prime}, 0\right) f\left(x_{2}^{\prime}, y_{2}^{\prime}, 0\right)<0
$$

Choosing $x_{3}$ that $0<f\left(x_{3}, 0,1\right) \leqq \frac{1}{4}+a$ we find that

and

$$
\left|d_{1}\right|^{-1} M_{+}(f) \leqq \frac{731}{3965} \cdot \frac{8}{3 a}\left(\frac{1}{4}+a\right)<\cdot 603
$$

$$
\left|d_{1}\right|^{-1} M_{+}(-f) \leqq \frac{1}{6} \cdot \frac{8}{3 a}\left(\frac{1}{4}+a\right)<\cdot 55 \text {. }
$$

(d) $\phi$ is equivalent to a form $a x^{2}+b x y+c y^{2}$ with $-\frac{5}{8} d_{1} \leqq a c<0$. In this case we take $a>0, c<0$ and

$$
f=(x+\lambda y+\mu z)^{2}-\left(a y^{2}+b y z+c z^{2}\right) .
$$

Choosing $x_{3}$ such that $\left|x_{3}+\mu\right| \leqq \frac{1}{2}$ and $x_{1}, \cdots, y_{2}^{\prime}$ is a similar way to that used in (c) we find that

$$
\begin{aligned}
& \left|d_{1}\right|^{-1} M_{+}(f) \leqq \frac{731}{3965} \cdot 4 a\left\{|c|+\frac{1}{4}\right\}\left|d_{1}\right|^{-1}, \\
& \left|d_{1}\right|^{-1} M_{+}(-f) \leqq \frac{1}{6} \cdot 4 a\left\{|c|+\frac{1}{4}\right\}\left|d_{1}\right|^{-1} .
\end{aligned}
$$

Thus as $\left|d_{1}\right|^{-1} \leqq 5 / 8 a|c|$ and $|c| \geqq \frac{3}{4}$ we find that

$$
\left|d_{1}\right|^{-1} M_{+}(f) \leqq 1462 / 2379 \text { and }\left|d_{1}\right|^{-1} M_{+}(-f)<\cdot 56
$$

Hence in each of the four possible cases we find that both $\left|d_{1}\right|^{-1} M_{+}(f)$ and $\left|d_{1}\right|{ }^{-1} M_{+}(-f)$ are bounded above by $1462 / 2379$.

Suppose now that $\phi$ is positive definite, in which case we may take $\phi$ to be the reduced form of its class satisfying

$$
0 \leqq b \leqq a \leqq c, a c \leqq \frac{1}{3}|D|
$$

We suppose, for the present, that in addition $f(x, y, 0)$ is not equivalent to a mul- 
tiple of any of

$$
x^{2}-5 / 2 y^{2}, x^{2}+k x y-y^{2} \quad(k=1,2,3 \text { or } 4) .
$$

By lemma 3 we have $a \geqq 2$ and so $c \geqq 2$. We choose $x_{3}$ such that

$$
\left|f\left(x_{3}, 0,1\right)\right| \leqq \begin{cases}c-1 ; & 2 \leqq c \leqq 2 \frac{1}{2} \\ \left(c-\frac{1}{4}\right)^{\frac{1}{1}} ; & c \geqq 2 \frac{1}{2} .\end{cases}
$$

We then choose $x_{1}, x_{1}^{\prime}, \cdots, y_{2}^{\prime}$ in a way similar to that used in (c) above to deduce that $M_{+}(f)$ and $M_{+}(-f)$ are bounded above

$$
\text { by } \frac{731}{3965} 4 a(c-1) \text { for } 2 \leqq c \leqq 2 \frac{1}{2} \text { and by } \frac{731}{3965} 4 a\left(c-\frac{1}{4}\right)^{\frac{1}{2}} \text { for } c \geqq 2 \frac{1}{2} \text {. }
$$

Since $a c \leqq 4 / 3\left|d_{1}\right|$ we have $\left|d_{1}\right|^{-1} M_{+}(f)$ and $\left|d_{1}\right|^{-1} M_{+}(-f)$ bounded above by $\frac{731}{3965} \frac{16}{3}\left(1-\frac{1}{c}\right)$ for $2 \leqq c \leqq 2 \frac{1}{2}$ and by $\frac{731}{3965} \cdot \frac{16}{3}\left(\frac{1}{c}-\frac{1}{4 c^{2}}\right)^{\frac{1}{2}}$ for $c \geqq 2 \frac{1}{2}$.

For the ranges of $c$ considered both expressions are a maximum at $c=2 \frac{1}{2}$ and these maxima are both $\frac{731}{3965} \cdot \frac{16}{5}<3 / 5$. Hence to complete the proof of the theorem it remains to consider the previously excluded possibilities for $f(x, y, 0)$. Since each of the excluded forms is equivalent to its negative we may consider the multiple to be positive.

Case 1. Suppose $f(x, y, 0)$ is equivalent to a multiple of $x^{2}-\frac{5}{2} y^{2}$, that is $(x+\lambda y)^{2}-a y^{2} \sim k\left(x^{2}-\frac{5}{2} y^{2}\right)$.

The lower bound of values of the left side lies between 1 and $1-\varepsilon$, while for the right side it is $k$. Thus $1-\varepsilon \leqq k \leqq 1$. The right side when multiplied by $2 k^{-1}$ has integral coefficients, so from the left side $2 k^{-1}, 4 k^{-1} \lambda$, and $2 k^{-1}\left(\lambda^{2}-a\right)$ are each integral. Hence $k=1$, while $4 \lambda$ and $2\left(\lambda^{2}-a\right)$ are integral. Comparing discriminants gives $a=\frac{5}{2}$, so $2 \lambda^{2}$ is integral. As we may take $|\lambda| \leqq \frac{1}{2}$ without loss of generality we have $\lambda=0$. Thus

where

$$
f(x, y, z)=(x+\mu z)^{2}-\left(\frac{5}{2} y^{2}+b y z+c z^{2}\right)
$$

$$
0 \leqq b \leqq \frac{5}{2} \leqq c,|D| \geqq \frac{15}{2} c .
$$

Now $(x+\mu z)^{2}-c z^{2}$ is not equivalent to a multiple of $x^{2}+x z-z^{2}$ as $c \geqq \frac{5}{2}$, so by lemma 1 there exist $x_{1}, x_{2}, z_{1}, z_{2}$ with $\left|x_{1} z_{2}-x_{2} z_{1}\right|=1$ and

$$
f\left(x_{1}, 0, z_{1}\right) f\left(x_{2}, 0, z_{2}\right) \leqq 3 c / 5 .
$$

By taking $f(1,1,0)=3 / 2$ or $f(2,1,0)=-3 / 2$ as necessary we find that $M_{+}(f)$, $M_{+}(-f)$ are bounded above by $\frac{9}{10} c$. Hence as $c \leqq \frac{8}{15}\left|d_{1}\right|$ we have $\left|d_{1}\right|^{-1} M_{+}(f)$, $\left|d_{1}\right|^{-1} M_{+}(-f)$ both bounded above by $12 / 25$. 
Case II. Suppose $f(x, y, 0)$ is equivalent to a multiple of $x^{2}+x y-y^{2}$. Arguing as in the previous case we may take

$$
f(x, y, z)=\left(x+\frac{1}{2} y+\mu z\right)^{2}-\left(\frac{5}{4} y^{2}+b y z+c z^{2}\right)
$$

with

$$
0 \leqq b \leqq 5 / 4 \leqq c,|D| \geqq \frac{15}{4} c .
$$

Barnes [1] has shown that for $c \geqq 2 \cdot 99$ we can choose $x_{3}$ such that

$$
\left|f(1,0,0) f(0,1,0) f\left(x_{3}, 0,1\right)\right|<\frac{4}{8 \cdot 9}\left|d_{1}\right| \text {. }
$$

He has also shown that for $2 \cdot 21(1-\varepsilon)^{2} \leqq c<2 \cdot 99$ we can choose $x_{1}, x_{3}, z_{1}, z_{3}$ with $\left|x_{1} z_{3}-x_{3} z_{1}\right|=1$ such that

$$
\left|f\left(x_{1}, 0, z_{1}\right) f(0,1,0) f\left(x_{3}, 0, z_{3}\right)\right|<\frac{4}{8 \cdot 87}\left|d_{1}\right| .
$$

Hence, replacing $f(0,1,0)=-1$ by $f(1,1,0)=1$ if necessary it is clear that $\left|d_{1}\right|^{-1} M_{+}(f)$ and $\left|d_{1}\right|^{-1} M_{+}(-f)$ are bounded above by $\frac{1}{2}$ for this range of $c$. By lemma 3 there are two further possibilities for $c$, namely:

(a) $c=2, f(x, 0, z) \sim k\left(x^{2}-2 y^{2}\right)$. In this case Barnes has shown that after a suitable transformation

$$
|f(1,0,0) f(0,1,0) f(1,0,1)|<(2 / 5+0(\varepsilon))\left|d_{1}\right|
$$

and so replacing $f(0,1,0)$ by $f(1,1,0)$ if necessary we find that $M_{+}(f)$ and $M_{+}(-f)$ are bounded above by $\frac{1}{2}\left|d_{1}\right|$ for suitable small $\varepsilon$.

(b) $c=5 / 4$. In this case Barnes has shown that $f \sim-Q_{1}$.

Case III. Suppose $f(x, y, 0)$ is equivalent to a multiple of $x^{2}+2 x y-y^{2}$ which is equivalent to $x^{2}-2 y^{2}$. Arguing as in case $\mathrm{I}$, we may take

with

$$
f(x, y, z)=(x+\mu z)^{2}-\left(2 y^{2}+b y z+c z^{2}\right)
$$

$$
0 \leqq b \leqq 2 \leqq c,|D| \geqq 6 c .
$$

We consider firstly the cases where $c \geqq 2 \cdot 21(1-\varepsilon)^{2}$.

(a) $2 \cdot 2<c<3 \frac{1}{4}-\varepsilon$. We choose $x_{3}$ such that $2 \frac{1}{4} \leqq\left(x_{3}+\mu\right)^{2} \leqq 4$ and $x_{3}^{\prime}$ such that $1 \leqq\left(x_{3}^{\prime}+\mu\right)^{2} \leqq 2 \frac{1}{4}$. Since $m(f) \geqq 1-\varepsilon$ we have

and

$$
0<f(1,0,0) f(1,1,0) f\left(x_{3}, 0,1\right) \leqq-(1-c)
$$

$$
4-c<f(1,0,0) f(1,1,0) f\left(x_{3}^{\prime}, 0,1\right)<0 .
$$


Now $d_{1}=2 c-\frac{1}{4} b^{2} \geqq 2 c-1$, so plainly

$$
\left|d_{1}\right|^{-1} M_{+}(f) \leqq(c-1) /(2 c-1)=\frac{1}{2}\left(1-(2 c-1)^{-1}\right)
$$

and

$$
\left|d_{1}\right|^{-1} M_{+}(-f) \leqq(4-c) /(2 c-1)=\frac{1}{2}\left(-1+7(2 c-1)^{-1}\right) .
$$

For the range of $c$ under consideration these bounds are at most $9 / 22,9 / 17$ respectively.

(b) $c \geqq 3 \frac{1}{4}-\varepsilon$. We choose $x_{3}$ such that

$$
\left|f\left(x_{3}, 0,1\right)\right| \leqq c-2 \frac{1}{4} \text { for } c \leqq 4 \frac{1}{4} \text { or }\left|f\left(x_{3}, 0,1\right)\right| \leqq\left(c-\frac{1}{4}\right)^{\frac{1}{2}} \text { for } c \geqq 4 \frac{1}{4} .
$$

Taking $f(1,0,0)=1$ and either $f(2,1,0)=2$ or $f(1,1,0)=-1$ we find that $\left|d_{1}\right|^{-1} M_{+}(f),\left|d_{1}\right|^{-1} M_{+}(-f)$ are bounded above by $2\left(c-2 \frac{1}{4}\right)(2 c-1)^{-1}$ for $c \leqq 4 \frac{1}{4}$ and $2\left(c-\frac{1}{4}\right)^{\frac{1}{2}}(2 c-1)^{-1}$ for $c \geqq 4 \frac{1}{4}$. For the allowable ranges of $c$, these both have a maximum of $\frac{8}{15}=\cdot 533 \cdots$ at $c=4 \frac{1}{4}$. Hence we find $\left|d_{1}\right|^{-1} M_{+}(f)$ and $\left|d_{1}\right|^{-1} M_{+}(-f)$ bounded above by $\frac{8}{15}$.

By lemma 3 it remains to consider the case

(c) $c=2, f(x, 0, z) \sim k\left(x^{2}-2 z^{2}\right)$. Without loss of generality we can take

$$
f(x, y, z)=x^{2}-\left(2 y^{2}+b y z+2 z^{2}\right)
$$

where $0 \leqq b \leqq 2$. Since $\phi(1,-1)=4-b$ we have by lemma 3 that either

$$
b=2 \text { or } b \leqq 4-2 \cdot 21(1-\varepsilon)^{2} .
$$

For $b=2$ we have

and

$$
f(1,1,0) f(1,0,1) f(1,1,-1)=-1=-\frac{1}{3}\left|d_{1}\right|
$$

$$
f(1,0,0) f(1,0,0) f(1,0,1)=1=\frac{1}{3}\left|d_{1}\right|,
$$

so for this form we certainly have $\left|d_{1}\right|^{-1} M_{+}(f)$ and $\left|d_{1}\right|^{-1} M_{+}(-f)$ both bounded above by $\frac{1}{3}$.

It remains to discuss the case $b \leqq 4-2 \cdot 21(1-\varepsilon)^{2}<1 \cdot 8$ for suitably small $\varepsilon$. Plainly $b \geqq 1-\varepsilon$ since

$$
f(2,1,-1)=b>-m(f) \text {. }
$$

Now $f(5,1,3)=5-3 b>-0 \cdot 4>-m(f)$ for small $\varepsilon$, so $5-3 b \geqq 1-\varepsilon$, i.e. $b \leqq 4 / 3+\frac{1}{3} \varepsilon$. But now $f(4,1,-3)=-4+3 b$ which is at most $\varepsilon$. Hence $-4+3 b \leqq-1+\varepsilon$, so $b \leqq 1+\frac{1}{3} \varepsilon$. Plainly, since $\left|d_{1}\right|=3 \frac{3}{4}+0(\varepsilon)$ and

$$
M_{+}(f) \leqq f(1,0,0) f(1,1,0) f(1,0,1)=1
$$

and 


$$
M_{-}(f) \leqq f(1,0,0) f(1,1,0) f(3,-2,1)=1+0(\varepsilon),
$$

we have $\left|d_{1}\right|^{-1} M_{+}(f)$ and $\left|d_{1}\right|^{-1} M_{+}(-f)$ both bounded above by $4 / 15+0(\varepsilon)$.

Case $I V$. Suppose $f(x, y, 0)$ is equivalent to a multiple of $x^{2}+k x y-y^{2}$ where $k=3$ or 4 . As usual we may take

with

$$
f=\left(x+\frac{1}{2}(4-k) y+\mu z\right)^{2}-\left(\left(1+\frac{1}{4} k^{2}\right) y^{2}+b y z+c z^{2}\right)
$$

$$
0 \leqq b \leqq 1+\frac{1}{4} k^{2} \leqq c .
$$

For $k=3$ we choose $x_{3}$ such that

or

$$
\left|f\left(x_{3}, 0,1\right)\right| \leqq c-2 \frac{1}{4} \text { for } c \leqq 4 \frac{1}{4}
$$

$$
\left|f\left(x_{3}, 0,1\right)\right| \leqq\left(c-3 \frac{1}{4}\right)^{\frac{1}{2}} \text { for } c \geqq 4 \frac{1}{4} .
$$

Taking $f(1,0,0)$ with either $f(1,0,0)=-1$ or $f(2,0,0)=3$ it is clear that $M_{+}(f), M_{+}(-f)$ are at most

$$
3\left(c-2 \frac{1}{4}\right) \text { for } c \leqq 4 \frac{1}{4} \text { and } 3\left(c-\frac{1}{4}\right)^{\frac{1}{2}} \text { for } c \leqq 4 \frac{1}{4} \text {. }
$$

Using the bound on $b$ to give a bound on $d_{1}$ we then find that $\left|d_{1}\right|^{-1} M_{+}(f)$ and $\left|d_{1}\right|^{-1} M_{+}(-f)$ are bounded above by

$$
\frac{12}{13}\left(c-2 \frac{1}{4}\right)\left(c-\frac{13}{16}\right)^{-1} \text { for } c \leqq 4 \frac{1}{4} \text { and } \frac{12}{13}\left(c-\frac{1}{4}\right)^{\frac{1}{2}}\left(c-\frac{13}{16}\right)^{-1} \text { for } c \geqq 4 \frac{1}{4} \text {. }
$$

In the allowable ranges for $c$, for $c=4 \frac{1}{4}$ each expression is a maximum of $384 / 715=\cdot 53 \cdots$.

For $k=4$, choosing $x_{3}$ such that $\left|f\left(x_{3}, 0,1\right)\right| \leqq\left(c-\frac{1}{4}\right)^{\frac{1}{2}}$, and taking $f(1,0,0)$ with either $f(2,1,0)=-1$ or $f(3,1,0)=4$ leads by a similar argument to the upper bound $8 \sqrt{ } 19 / 75=\cdot 46 \cdots$ for $\left|d_{1}\right|^{-1} M_{+}(f)$ and $\left|d_{1}\right|^{-1} M_{+}(-f)$. This completes case IV and so theorem 1 is proven.

Remarks. It would be possible to define quantities such as $M_{+++}, M_{++-}$, $M_{+--}$and $M_{-\ldots}$ for a ternary quadratic form, where e.g. $M_{++-}$is the lower bound of

$$
\left|Q\left(x_{1}, y_{1}, z_{1}\right) Q\left(x_{2}, y_{2}, z_{2}\right) Q\left(x_{3}, y_{3}, z_{3}\right)\right|
$$

over integral $x_{1}, \cdots, z_{3}$ satisfying (1.1) such that two of the values are positive (or non-negative ) and the other value is negative (or non-positive). The constant $\frac{2}{3}$ would have to be increased for some of these problems, since for example the form

$$
Q_{5}(x, y, z)=3 x^{2}+8\left(x y+y^{2}-z^{2}+y z\right)
$$


has all its negative values at most -5 and its positive values at least 3 . Thus $M_{+--}\left(Q_{5}\right) \geqq \frac{75}{112}|d|$. Indeed

$$
Q_{6}(x, y, z)=x^{2}+3\left(x y+y^{2}-z^{2}+y z\right)
$$

has $M_{+--}\left(Q_{6}\right) \geqq \frac{8}{9}|d|$. For discussion of these forms see lemmas $2.6,2.7$ of [6].

\section{References}

[1] E. S. Barnes, 'On Indefinite Ternary Quadratic forms', Proc. Lond. Math. Soc. (3) 2 (1952), 218-233.

[2] E. S. Barnes, 'The Minimum of the Product of two Values of a Quadratic Form III', Proc. Lond. Math. Soc. (3) 1 (1951), 415-434.

[3] L. E. Dickson, Studies in the Theory of Numbers (Chicago, 1930).

[4] H. Blaney, 'Indefinite Quadratic Forms in $n$ Variables', J. Lond. Math. Soc. 23 (1948), 153-160.

[5] A. Oppenheim, 'Values of Quadratic Forms', Quart. J. Math. (Ox.) (2) 4 (1953), 54-59.

[6] R. T. Worley, 'Asymmetric Minima of Indefinite Ternary Quadratic Forms', J. Austral. Math. Soc. 7 (1967), 191-238. 